\title{
NOTE ON CURRENCY
}

In the text the terms '£ sterling' and '£ currency' have been used. The former refers to British currency, the latter to the currencies of the individual Caribbean colonies.

[xii ] 